\title{
DÉTERMINATION RAPIDE DE LA CONDUCTIVITÉ THERMIQUE D'ÉCHANTILLONS FORTEMENT RÉSISTIFS. APPLICATION AU DITELLURURE DE MOLYBDẼNE
}

\author{
M. ZOAETER (*), A. CONAN et P. GUILLON
}

Laboratoire de Physique du Métal et d'Electronique, B.P. 1044, Nantes, France

(Reçu le 4 mars 1977, révisé le 27 avril 1977, accepté le 17 mai 1977)

\begin{abstract}
Résumé. - Nous proposons une méthode originale de mesure de conductivité thermique, basée sur l'étude du phénomène de résistance négative observé sur des échantillons de type semi-conducteur. Cette méthode est utilisée sur des échantillons de dichalcogénures de molybdène stœechiométrique ou non. Les résultats obtenus sont confrontés aux valeurs trouvées par une mesure classique et les écarts entre ces mesures n'excèdent jamais $7 \%$. La variation de la conductivité thermique en fonction de l'écart à la stœchiométrie présente une anomalie analogue à celle déjà observée sur les courbes de conductivité électrique et du P.T. E.
\end{abstract}

\begin{abstract}
A new method for the rapid determination of the thermal conductivity of semiconducting-type samples is described. This method, based on the non-ohmic behaviour of these compounds, has been tested on three stœchiometric or non-stœechiometric molybdenium dichalcogenides. The results are compared with the values found by a conventional method. The differences observed between these measurements are less than $7 \%$. A singularity of the thermal conductivity, plotted as a function of the composition $\times$, has been found.
\end{abstract}

1. Introduction. - La conductivité thermique des chalcogénures est faible à basse température $(K \simeq 0,02 \mathrm{~W} / \mathrm{cm} . \mathrm{K})$. Elle reste sensiblement constante jusqu'à ce que la contribution électronique intervienne de façon significative. Perron [1] a montré que la composante électronique de la conductivité thermique des alliages Se-Te obéit à la loi de Wiedemann-Franz avec un nombre de Lorenz de l'ordre de

$$
2,45 \times 10^{-8} \mathrm{~W} \Omega / \mathrm{K}^{2} .
$$

Dans deux articles précédents [2, 3], nous avons étudié la conductivité électrique et le pouvoir thermoélectrique (P. T. E.) de composés stœchiométriques ou non de type $\mathrm{MoTe}_{2-x}(0<x \leqslant 0,4)$. Ces études, effectuées sur une large étendue de température (120$500 \mathrm{~K}$ ), se sont montrées suffisantes pour déterminer les divers mécanismes qui participent aux phénomènes de transport et de proposer un modèle de bande pour ces composés. Afin de mieux caractériser ces matériaux et de compléter cette étude, nous avons mis au point une méthode originale de mesure de conductivité thermique.

(*) Attaché de recherche au C. N. R. S. Liban.
Les méthodes de mesure de conductivité thermique peuvent se classer en deux catégories : les méthodes statiques et les méthodes dynamiques. Les premières ont l'avantage de la précision de mesure, les secondes de la rapidité. Nous avons réalisé une cellule de mesure dynamique de conductivité thermique et confronté nos résultats à ceux obtenus par une méthode statique. Cette étude s'est effectuée sur des échantillons dont la méthode de préparation a été décrite précédemment [2] Ils se présentent sous forme de disques de poudres fortement compactées, de diamètre $8 \mathrm{~mm}$ et d'épaisseur $2 \mathrm{~mm}$. La compacité des échantillons est de l'ordre de 60 à $70 \%$. Lors du compactage, les cristallites ont tendance à orienter leurs axes c parallèlement à la direction de la pression. Les mesures se font dans la direction de l'axe du cylindre qui est confondu avec l'axe c.

2. Méthodes de mesure. - Les méthodes de mesure dynamique de conductivité thermique sont basées sur le principe suivant : un échantillon, dont les extrémités sont maintenues à température constante, est parcouru par un courant électrique constant. Un régime stationnaire s'établit rapidement et il est alors possible de relier la distribution de température le long de l'échan- 
tillon au rapport des conductivités électrique et thermique. Cette méthode, mise au point par Kohlrausch, permet de déterminer avec une bonne précision, la conductivité thermique d'échantillons de type métallique pour lesquels la variation de résistivité est linéaire en température. Elle a récemment été modifiée [4] pour mesurer la conductivité thermique du tungstène. Nous avons adapté cette dernière aux échantillons $\mathrm{MoTe}_{2-x}$ fortement résistifs.

Dans un article récent [5] nous avons étudié la distribution de température le long de barreaux fortement résistifs lorsque leurs extrémités sont maintenues à température constante $T_{0}$ et qu'ils sont parcourus par un courant constant $I$ : dès que le courant dépasse une valeur critique $I_{\text {cr }}$, à laquelle correspond une valeur de la tension $U_{\mathrm{cr}}$, les échantillons présentent un phénomène de résistance dynamique négative. L'expression théorique de la tension critique est, en utilisant les notations de [6], donnée par:

$$
\begin{aligned}
& U_{\text {cr }}=1,88\left[\frac{k T_{0}^{2}}{\bar{E}\left(T_{0}\right)} \frac{K\left(T_{0}\right)}{\sigma\left(T_{0}\right)}\right]^{1 / 2} \\
& \bar{E}\left(T_{0}\right)=\frac{\sigma_{1}\left(T_{0}\right) E_{1}+\sigma_{2}\left(T_{0}\right)\left[E_{2}+k T_{0}\right]}{\sigma_{1}\left(T_{0}\right)+\sigma_{2}\left(T_{0}\right)}
\end{aligned}
$$

où $k$ est la constante de Boltzmann. $K$ est la conductivité thermique. $\sigma_{1}$ est la conductivité électrique due aux trous de la bande de valence dont l'énergie d'activation est $E_{1}=E_{\mathrm{F}}-E_{\mathrm{v}} . \sigma_{2}$ est la conductivité due aux électrons des états localisés dans la queue de bande de conduction dont l'énergie d'activation est

$$
E_{2}=E_{\mathrm{A}}-E_{\mathrm{F}}+W
$$

$\left(E_{\mathrm{A}}-E_{\mathrm{F}}\right.$ est la différence d'énergie entre le bas de queue de bande et le niveau de Fermi, $W$ est l'énergie nécessaire pour effectuer un saut).

Plusieurs autres études théoriques $[6,7,8,9]$ ont été effectuées sur des semiconducteurs de formes variées. Toutes conduisent à des relations de proportionnalité de la forme :

$$
U_{\mathrm{cr}}=A\left[K / \frac{\mathrm{d} \sigma}{\mathrm{d} T}\right]_{T=T_{0}}^{1 / 2}
$$

où $A$ est une constante qui ne dépend que de la géométrie de l'échantillon. En particulier, Stocker et coll. [7] ont montré que :

- dans le cas d'un cylindre de rayon $R$ et de génératrice $L$, le coefficient $A$ est donné par : $A=L \sqrt{2} / R$.

Ces expressions montrent que, si $\mathrm{d} \sigma / \mathrm{d} T$ est proportionnel à $\sigma$, la mesure de la tension critique permet d'accéder au rapport des conductivités thermique et électrique et en conséquence à la valeur de la conductivité thermique $K$ de l'échantillon.

2.1 Cellule de mesure. - Afin d'éviter les fuites thermiques par conduction latérale et convection, la cellule de mesure est placée dans une enceinte à vide.
Cette cellule n'est autre que celle qui est utilisée pour la détermination à haute température du P. T. E. et de la conductivité électrique de nos échantillons. Ceci présente l'avantage de réduire le temps de manipulation : dès que la température de l'échantillon est stabilisée, il est possible d'effectuer consécutivement les trois mesures. Un schéma synoptique du montage est donné figure 1. L'échantillon (1) est comprimé entre deux

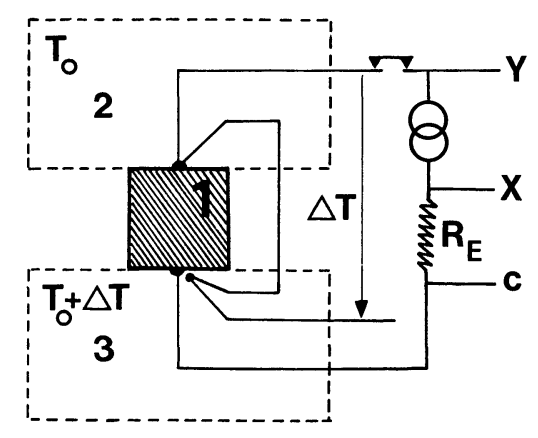

Fig. 1. - Schéma de la cellule de mesure. [Block diagram of equipment]

blocs de cuivre autour desquels sont enroulés des fils de chauffage en thermocoax (2) et (3). Le premier enroulement (2) constitue un four qui est régulé pour maintenir la face supérieure de l'échantillon à température constante $T_{0}$. Le second enroulement permet de faire varier la différence de température entre les deux faces de l'échantillon. Cette différence de température est mesurée à l'aide d'un thermocouple monté en différentiel. Lors d'une mesure de conductivité thermique, le second enroulement permet de maintenir la température de la face inférieure de l'échantillon à $T_{0}$ et le thermocouple monté en différentiel permet de la contrôler. Un générateur de courant, placé en série avec une résistance étalon, alimente l'échantillon par l'intermédiaire de deux fils de cuivre. Les tensions qui apparaissent aux bornes de la résistance (proportionnelle au courant) et aux bornes de l'échantillon sont envoyées sur les voies $\mathrm{X}$ et $\mathrm{Y}$ d'une table traçante La caractéristique courant-tension de l'échantillon est déterminée et la tension critique est mesurée. La valeur de la conductivité thermique peut alors être calculée à l'aide de la relation (1).

2.2 Influence De LA PRESSION DE CONTACT. Nous avons signalé [3] qu'il est difficile de donner avec précision la valeur de la conductivité électrique $\sigma$ des composés étudiés car cette dernière dépend de la pression exercée sur l'échantillon. En effet, ces derniers ne sont pas frittés mais constitués de poudres très fines fortement comprimées. Lors de la préparation, la pression exercée est la même pour tous les échantillons $\left(10^{3} \mathrm{bar} / \mathrm{cm}^{2}\right)$. Ces derniers présentent donc tous la même compacité. Dans la cellule de mesure, l'échantillon est serré entre deux blocs de cuivre à l'aide d'un ressort dont on peut faire varier la tension. La pression qui s'exerce alors est fonction de la tension appliquée. 
il existe donc une résistance additionnelle de contact entre les grains qui diminue lorsque la pression augmente. Ceci se traduit par une modification des termes préexponentiels des conductivités. On a cependant constaté que les courbes représentatives de $\ln \sigma$ en fonction de $10^{3} / \mathrm{T}$ tracées pour diverses pressions appliquées au même composé se déduisent les unes des autres par de simples translations. La valeur de l'énergie d'activation $\bar{E}$ est donc indépendante de la pression. De plus, le phénomène de résistance négative a lieu à partir du moment où la puissance électrique four-

$\begin{array}{cccc}\text { Pression } & & \mathrm{MoTe}_{2} \\ \overline{-} & \overline{U_{\text {cr }}} & R\left(T_{0}\right) & U_{\text {cr }}^{2} / R \\ P_{1} & 54,5 & 612,7 & 4,85 \\ P_{2} & 53,4 & 587,9 & 4,85 \\ P_{3} & 50,5 & 524,7 & 4,86\end{array}$

$U_{\text {cr }}$ est mesurée en volts, $R\left(T_{0}\right)$ en ohms et la puissance en watts.

Ce tableau montre que quelle que soit la pression exercée, la puissance critique dissipée est constante à mieux de $7 \%$ près. La valeur calculée de la conductivité thermique dépendra donc peu de la pression exercée. Ce résultat était prévisible car la conductivité électrique est un phénomène essentiellement dû aux trous de la bande de valence et aux électrons localisés dans la queue de la bande de conduction, tandis que la contribution la plus importante pour la conductivité thermique provient des atomes du réseau.

Remarques. - La valeur de la section $S$ est la même pour tous les composés étudiés car tous sont comprimés dans la même matrice. Seule l'épaisseur $2 e$ varie légèrement d'un échantillon à l'autre car elle dépend de la quantité de matériaux utilisée. L'expression (3) s'écrit alors :

$$
K\left(T_{0}\right)=1,126 e \frac{\bar{E}}{k T_{0}^{2}} \frac{U_{\mathrm{cr}}^{2}}{R} \mathrm{~W} / \mathrm{cm} \cdot \mathrm{K} .
$$

La valeur de $\bar{E}$ est, soit directement déterminée sur la caractéristique $\ln \sigma=f\left(10^{3} / T\right)$, soit calculée à l'aide des valeurs théoriques données dans [3, 5].

2.3 Comparaison AVEC LES MÉTHODES ClASSIQUES. - Les mesures de conductivité thermique des solides reposent sur des principes établis depuis longtemps et la majorité des techniques expérimentales utilisées [10 à 13] est basée sur l'équation de Fourier : un gradient de température est établi dans le sens de la longueur de l'échantillon en introduisant un flux de chaleur $\Phi$ par l'une de ses faces et en l'évacuant par l'autre face. S'il n'existe pas de pertes de chaleur latérales, la conductivité thermique $K$ en régime stationnaire s'exprime par :

$$
K=\frac{\Phi}{S}\left(\frac{\mathrm{d} T}{\mathrm{~d} x}\right)^{-1}
$$

où $S$ est la section de l'échantillon. nie à l'échantillon devient supérieure à celle que celui-ci peut dissiper. Or la relation (1) peut s'écrire :

$$
\frac{U_{\mathrm{cr}}^{2}}{R\left(T_{0}\right)}=1,767 \frac{S}{e} \frac{k T_{0}^{2}}{\bar{E}} K\left(T_{0}\right)
$$

où $S$ est la section de l'échantillon et $2 e$ son épaisseur. Nous avons calculé la puissance dissipée dans trois échantillons de comportement semiconducteur pour des pressions appliquées, par l'intermédiaire du ressort, $P_{1}<P_{2}<P_{3}$. Les résultats obtenus sont résumés sous forme de tableau ci-dessous :

\begin{tabular}{cccccc}
\multicolumn{3}{c}{ Composé $^{\text {MoTe }_{1,98}}$} & & \multicolumn{3}{c}{$\mathrm{MoTe}_{1,97}$} \\
$U_{\text {cr }}$ & $R\left(T_{0}\right)$ & $U_{\text {cr }}^{2} / R$ & $U_{\text {cr }}$ & $R\left(T_{0}\right)$ & $U_{\text {cr }}^{2} / R$ \\
$\overline{44,5}$ & - & - & - & - & - \\
38,9 & 261,5 & 5,75 & 34,4 & 208,3 & 5,68 \\
37,8 & 246,8 & 5,78 & 32,4 & 185,8 & 5,65 \\
& & 5,79 & 29,9 & 157,6 & 5,67
\end{tabular}

La méthode que nous avons utilisée est une méthode comparative. Un schéma de la cellule de mesure est donné figure 2. L'échantillon (7) est comprimé entre

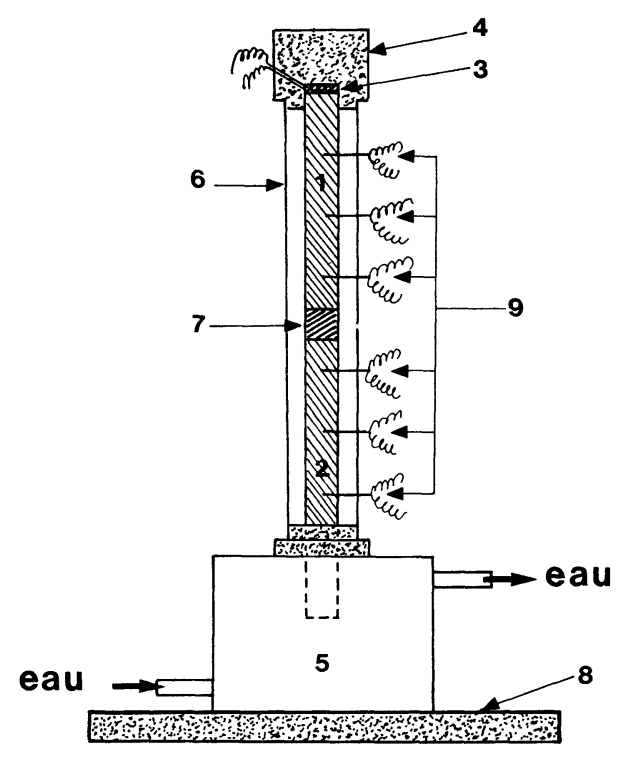

Fig. 2. - Cellule de mesure de la conductivité thermique : 1 et 2 : deux cylindres de cuivre ; $3:$ petit four ; 4 : isolant thermique ; 5 : masse de cuivre; 6 : écran thermique ; 7 : échantillon; $8:$ isolant $; 9:$ thermocouples.

[Schematic diagram of the experimental arrangement : 1.2. Copper rods ; 3 . Heater ; 4 . Thermal insulator ; 5 . Copper isothermal block; 6. Guard tube ; 7. Sample ; 8. Insulation ; 9. Thermocouples]

deux cylindres de cuivre (1) et (2) de même section que l'échantillon. Trois thermocouples, régulièrement espacés, plongent dans chacune des pièces en cuivre et servent à mesurer et à contrôler la linéarité du gradient de température le long de ces derniers. Un petit four (3), enroulé à plat en spirale, est soudé à l'extrémité supérieure du premier cylindre (1). L'extrémité inférieure $\mathrm{du}$ second cylindre (2) est placée dans une masse de cuivre (5) dont la température est régulée par une 
circulation d'eau. Afin de limiter les pertes thermiques latérales par conduction et convection, l'ensemble est placé dans une enceinte à vide.

$\mathrm{Si}$ les résistances thermiques de contact entre blocs de cuivre et échantillon sont négligeables, le rapport des gradients de température qui existent dans les pièces en cuivre et l'échantillon est dans le rapport inverse de leurs conductivités thermiques. Or la conductivité thermique du cuivre est connue avec une grande précision $(0,7 \%)$ [12] : elle varie linéairement avec la température et est donnée par :

$$
K_{\mathrm{Cu}}=4,1883-0,6174 \times 10^{-3} \mathrm{~T} \mathrm{~W} / \mathrm{cm} . \mathrm{K} .
$$

Le gradient de température dans les pièces de cuivre est mesuré ; celui qui existe dans l'échantillon se détermine graphiquement. Les températures indiquées par les thermocouples qui plongent dans les blocs de cuivre sont reportées en fonction de la position de ces derniers. L'intersection des droites obtenues, avec les parallèles à l'axe des températures aux abscisses qui correspondent à la position des faces de l'échantillon donne la différence de température entre ces faces.

3. Résultats. - Les mesures classiques de conductivité thermique sont faites dans une faible étendue de température $(300-330 \mathrm{~K})$ et les résultats sont reportés figure 3. La variation de $K$ est dans ce domaine sensi-

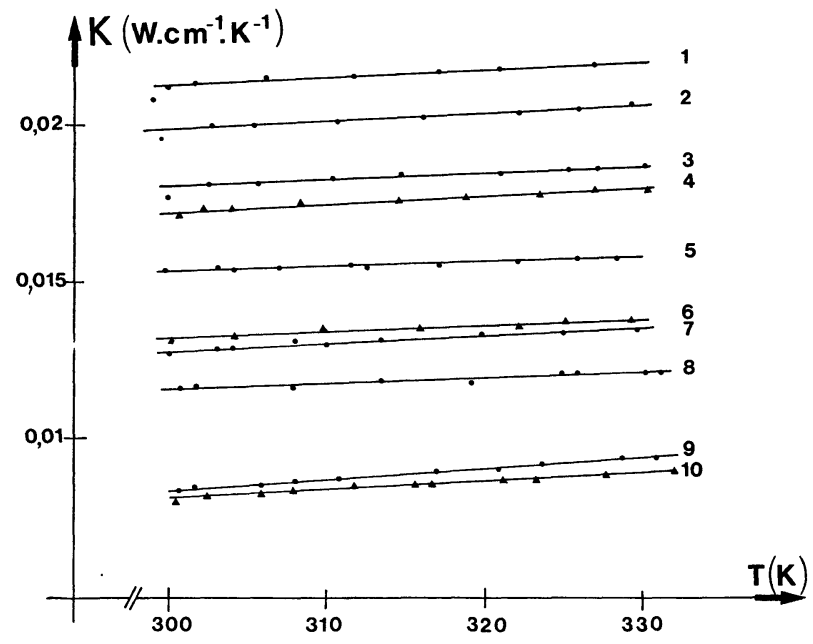

FIG. 3. - Variation de la conductivité thermique des composés $\mathrm{MoTe}_{2-x}$ en fonction de la température (300-330 K).
1: MoTe $_{2} 4:$ MoTe $_{1,93} 7:$ MoTe $_{1,95}$
$2: \mathrm{MoTe}_{1,91} 5: \mathrm{MoTe}_{1,98} \quad 8: \mathrm{MoTe}_{1,96}$
$3: \operatorname{MoTe}_{1,94} 6: \mathrm{MoTe}_{1,80} 9: \mathrm{MoTe}_{1,70}$
$10: \mathrm{MoTe}_{1,60}$

[Temperature variation of the thermal conductivity of $\mathrm{MoTe}_{2-x}$ compounds]

blement linéaire en température. La valeur de la conductivité thermique est faible et du même ordre de grandeur que celle observée sur les autres dichalcogénures, sa variation en température est peu significative dans le domaine de température étudié.

La variation de la conductivité thermique en fonc- tion de l'écart à la stœchiométrie (Fig. 4) présente une anomalie analogue à celle observée sur les courbes de conductivité électrique et de P. T. E. [1, 2] : lorsque l'écart à la stœchiométrie croît, la conductivité ther-

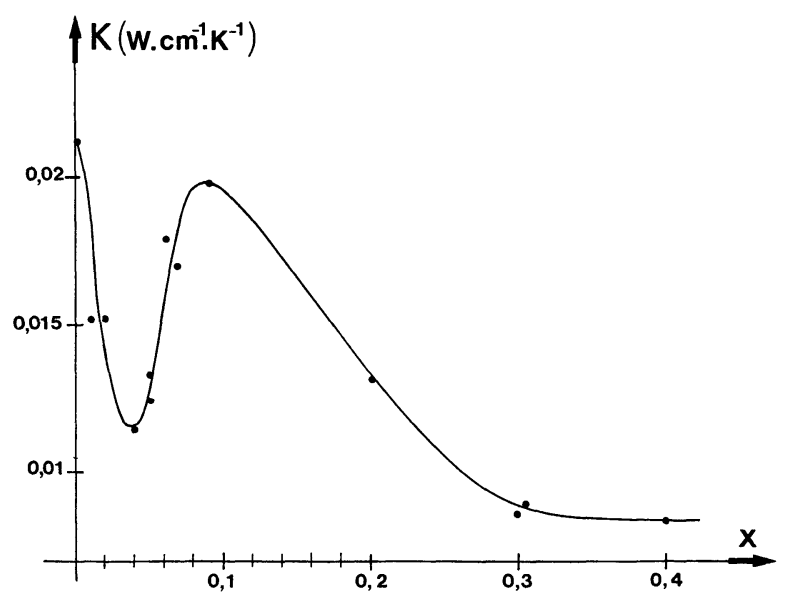

FIG. 4. - Variation de la conductivité thermique en fonction de l'écart à la stœchiométrie des composés $\mathrm{MoTe}_{2-x}$.

[Variation of the thermal conductivity as a function of composition for various non stœchiometric compounds $\mathrm{MoTe}_{2-x}$.]

mique décroît, passe par un minimum pour $\mathrm{MoTe}_{1,96}$ pour croître à nouveau jusqu'à la limite du domaine d'existence de la phase $\mathrm{MoTe}_{2}$. Dans le domaine diphasé, la conductivité thermique décroît lentement.

La comparaison entre les valeurs trouvées par une mesure classique de conductivité thermique et les valeurs calculées à partir de l'équation (4) a été faite à température ambiante. Les résultats obtenus sont donnés dans le tableau ci-dessous pour trois composés de comportement semiconducteur :

\begin{tabular}{llcl}
\multicolumn{1}{c}{ Composé } & $\mathrm{MoTe}_{2}$ & $\mathrm{MoTe}_{1,98}$ & $\mathrm{MoTe}_{1,97}$ \\
$K$ (classique) & $\overline{-}$ & - & - \\
$K$ donné par (4) & 0,0195 & 0,015 & 0,0126 \\
0,014 2 & 0,012
\end{tabular}

$K$ est exprimé en $\mathrm{W} / \mathrm{cm} . \mathrm{K}$.

Ce tableau montre que les valeurs trouvées par la méthode dynamique proposée sont légèrement inférieures à celles de la méthode classique, les écarts n'excédant pas 7 à $8 \%$. Cet écart serait accentué si l'on tenait compte des résistances thermiques additionnelles de contact entre échantillons et blocs de cuivre qui, bien que réduites au minimum par de la graisse conductrice, interviennent lors de la mesure classique.

On peut évaluer l'élévation moyenne de température dans l'échantillon : par raison de symétrie, la température maximale $T_{\mathrm{m}}$ dans l'échantillon dont les extrémités situées aux abscisses $-l$ et $+l$ sont maintenues à température constante $T_{0}$, s'obtient à l'abscisse 0 . Si on admet que la variation de température est linéaire dans l'échantillon, l'élévation moyenne de température est $\overline{\Delta T}=\left(T_{\mathrm{m}}-T_{0}\right) / 2$. Considérons la moitié de l'échantillon comprise entre 0 et $l$ : la puis- 
sance électrique qui y est dissipée (VI/2) est liée à l'élévation moyenne de température par la relation :

$\overline{\Delta T}=R_{\mathrm{T}}\left(\frac{V I}{2}\right)$

où $R_{\mathrm{T}}$ est la résistance thermique $\left(\frac{1}{K} \frac{l}{2 S}\right)(V I)$.

On trouve ainsi $\overline{\Delta T}=25 \mathrm{~K}$ pour $\mathrm{MoTe}_{2}$.

Un calcul exact, tenant compte de la distribution réelle de température conduit à $\overline{\Delta T}=26 \mathrm{~K}[5]$. La valeur de la conductivité thermique calculée est donc une valeur moyenne entre $T_{0}$ et $T_{\mathrm{m}}$.

La connaissance des coefficients de transport $S, \sigma$ et $K$ permet de calculer le facteur de mérite $Z=S^{2} \sigma / K$ de nos échantillons. Les valeurs trouvées sont très faibles bien que le rapport $S^{2} / K$ soit du même ordre de grandeur que celui qu'on observe sur de bons thermoéléments $\left(S^{2} / K\right)=8,4 \times 10^{-6}$ pour $\mathrm{MoTe}_{2}$ à comparer à $2,76 \times 10^{-6}$ de $\mathrm{Bi}_{2} \mathrm{Te}_{3}$.

Ceci est dû à la valeur élevée de la résistivité des échantillons. Pour améliorer ce facteur, il serait nécessaire de travailler sur des échantillons monocristallins et de doper ces derniers.

4. Conclusion. - Les résultats obtenus montrent que la méthode de mesure proposée permet de mesurer la conductivité thermique d'échantillons fortement semiconducteurs avec une bonne reproductibilité et une précision qui entre dans la limite des erreurs expérimentales. On ne peut toutefois en affirmer la précision absolue pour un échantillon dont la variation de conductivité thermique en fonction de la température serait importante (dans les calculs $K$ est supposé constant pour une faible variation de température). Cette méthode est comparable à celle proposée par [14] : elle présente la même facilité de mesure et ne nécessite pas un appareillage très performant, tout en ayant une bonne précision et contrairement aux méthodes statiques où il faut attendre longtemps pour stabiliser le flux thermique à travers l'échantillon, le régime stationnaire s'établit rapidement. La durée de mesure est de l'ordre d'une demi-heure pour la méthode statique (du fait de la valeur élevée de la résistance thermique) et n'est que quelques minutes pour la méthode proposée. L'étude des variations de conductivité thermique en fonction de l'écart à la stœchiométrie montre l'existence d'un minimum pour le composé $\mathrm{MoTe}_{1,96}$. Ce minimum, déjà trouvé dans les courbes de conductivité électrique et de P. T. E., est en pourcentage beaucoup moins marqué pour la conductivité thermique, ce qui prouve que les vibrations du réseau sont en grande partie responsables du phénomène de conduction. Ce minimum pourrait correspondre à une structure lacunaire ordonnée où les lacunes se localiseraient au sommet de chaque hexagone d'ordre 4 [15]. La présence d'une telle structure permettrait d'expliquer le passage de l'état semiconducteur de $\mathrm{MoTe}_{2}$ à l'état semi-métallique du $\mathrm{MoTe}_{1,96}$.

Remerciements. - Les auteurs remercient M. le Professeur J. P. Bardon de l'Université de Nantes pour les fructueuses discussions que nous avons eues.

\section{Bibliographie}

[1] Perron, J. C., Phys. Lett. A32 (1970) 169, Phys. lett. A32 (1970) 169.

[2] Conan A., Goureaux G., Zoaeter M., J. Phys. Chem. Solids 36 (1975) 315.

[3] Conan, A., Zoaeter, M., Goureaux, G., J. Physique 37 (1976) 1233.

[4] Jain, S. C., Bansal N. K., Sinha V., J. Phys. D. Appl. Phys. 8 (1975) 354.

[5] Conan, A., Zoaeter M., Goureaux, G., Phys. Stat. Sol. (a) 38505 (1976)

[6] Kroll, D. M., Phys. Rev. 9 (1974) 1669.
[7] Stocker, H. J., Barlow, C. A., J. Non-Cryst. Sol. 4 (1970) 523.

[8] Croitoru, N., Vescan, L., Popescu, C., Lazarescu, H., J. Non-Cryst. Sol. 4 (1970) 493.

[9] Chen, H. S., Wang, T. T., Phys. Stat. Sol (a) 2 (1970) 79.

[10] Moss, H., Rev. Sci. Instrum. 26 (1955) 3.

[11] Laubitz, M. J., Can. J. Phys. (1967) 45.

[12] Laubitz, M. J., Mc Elroy, D. L., Metrologia 7 (1971) 1.

[13] Whitsett, C. R., Nelson, D. A., Phys. Rev. B 5 (1972) 8.

[14] Pegna, G., Congiu, A., Revue Phys. Appl. 10 (1975) 177.

[15] Guillon, P., Conan, A., Zoaeter, M. et Goureaux, G., C. R. Hebd. Séan. Acad. Sci. (à paraître). 